\title{
A Time of Challenge and Growth: A Qualitative Study of Newly Qualified Nurses' Experience
}

\author{
Kai-Yu Tseng ${ }^{1}$, Hsing-Chi Hsu ${ }^{2 *}$

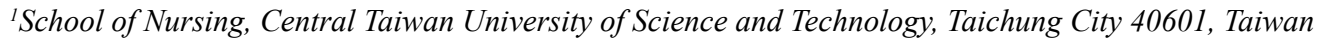 \\ ${ }^{2}$ Department of Public Health, China Medical University, Taichung, No.91 Hsueh-Shih Road,Taichung, Taiwan
}

\section{Article Details \\ Article Type: Research Article \\ Received date: $16^{\text {th }}$ October, 2017 \\ Accepted date: $08^{\text {th }}$ January, 2018 \\ Published date: $15^{\text {th }}$ February, 2018}

"Corresponding Author: Hsing-Chi Hsu, Department of Public Health, China Medical University, Taichung, No.91 HsuehShih Road, Taichung, Taiwan E-mail: chiqueens@gmail.com

Citation: Tseng KY, Hsu H-C (2018) A time of challenge and growth: a qualitative study of newly qualified nurses' experiences. J Comp Nurs Res Care 3: 121. doi: https://doi.org/10.33790/jenrc1100121.

Copyright: $\left({ }_{2} 2018\right.$, This is an open-access article distributed under the terms of the Creative Commons Attribution License 4.0, which permits unrestricted use, distribution, and reproduction in any medium, provided the original author and source are credited.

\begin{abstract}
Background: In any country the transition from student to Registered Nurse is a rewarding but challenging and stressful time. Nurses terminating employment within one year of qualification have been reported as $58 \%$ in Taiwan. Therefore, the experience of NQNs' transition from the role of student to qualified nurse after being employed in their first posts in their nursing careers in Taiwan is worthy of research.
\end{abstract}

Purpose: To explore experiences of newly qualified nurses (NQNs) during the first twelve months of their career in Taiwan.

Methods: A primarily cross-sectional qualitative research design was used. These were a purposive sample of $28 \mathrm{NQNs}$ during the first 12 months as Registered Nurses. Semi-structured interviews were undertaken with groups of participants. Data analysis was undertaken within the Thematic Content Analysis Framework.

Results: Four main themes of newly qualified nurses' experiences were identified: Perceptions of becoming a nurse; Reality challenge; Developing competence; and Interpersonal relationships.

Conclusion: NQN's experiences in clinical practice during the first twelve months employment sound very like a reality challenge. Successful transition was influenced by personal, social and environmental work factors. NQNs identified that their new role was based on what they evaluated as limited knowledge and skills. They addressed the importance of interpersonal relationship influenced their transition experiences. The findings have implications for education of student nurses and their support during transition to the role of qualified nurses. This requires a coordinated response from nursing schools, hospitals and government to increase those who adapt to their new roles and develop successful nursing careers.

Keywords: Newly Qualified Nurse, Role Transition, Qualitative Research

\section{Introduction}

Pre-registration nurse education, at any academic level or country, aims to prepare a student to become a competent qualified nurse and a valuable part of the nursing workforce. Across all models of preregistration nurse education many newly qualified nurses (NQNs) find the transition from student to qualified nurse is a title change, but also an adjustment to the new role[1-3].
Transition is an important process and occurs several times during people's lives. However, transition is not an event, but rather multiple psychological processes involving people going through the process of change. It is psychological and stems from a variety of life events and situations, developmental and socio-cultural situations brought about by the change [4-7].

Bridges [4] has developed "Managing Transition" with three phases of response to transition, starting with an ending and finishing with a beginning, and he suggested that in a different situation, people need to relinquish the old way and enter the new situation. Every beginning ends something. The first transitional phase, The Ending, means leaving one's previous state and entering the transition. 'When endings occur, people get angry, sad, frightened, depressed, and confused' [8]. The second transitional phase, The Neutral Zone, is both a dangerous and an opportune place because it is easy for people to become polarized. This last phase is a rebirth or reinvention of the self and the destination is discovery of a new sense of purpose.

"Managing Transition" framework describes the relations between time and the proportion of psychological activities taking place inside the individual during the transition period [4]. "Managing Transition" framework provides a useful and suitable guide to research NQNs experience in the first twelve months when they go through transition from student nurses to NQNs in Taiwan. In doing so it facilitates understanding of the mean- ing, process and consequences of transition of NQNs.

Nurses experience stress, value conflict and anxiety on transition to the Registered Nurse (RN) role, described as "reality shock" [9]. More recently Duschscher [10] proposed stages of "Transition Shock' and 'Transition Crisis' that new registrants must negotiate for successful transition. Andersson and Edberg [1] described new registrants as 'Rookies' with difficulty in independent task management. Transition from student to qualified nurse is often seen as challenging and stressful. International research from Ireland [1], Australia [12,13] and the United Kingdom [14] identify difficulties in adapting to the NQNs' role. However, the first year can also include growth and development as NQNs successfully become confident qualified nurses [15].

Researchers reported organizational and managerial skill deficits, 
clinical skills deficits and dealing with new situations as major factors causing stress [11]. They used phenomenology to explore NQNs' lived experiences during the first six months after qualifying in Ireland. They reported that NQNs identified deficits in clinical skills causing scared and nervous feelings. Also important were interpersonal relationships, obeying covert rules, lack of support and poor nursing role models $[1,16]$.

NQNs in Taiwan experience high clinical workloads and major differences between nursing education and clinical reality. The total numbers of nurses working in Taiwan in 2006 were 153,453 and 142 Taiwanese hospitals reported $28 \%$ of nurses terminating employment annually; among these, $58 \%$ of NQNs resigned within one year with $67 \%$ leaving because of pressure to adapt [17]. The high turnover of hospital nurses is linked to perception of a short nursing career, personal burnout, client-related burnout, poor workplace justice, and shorter work tenure as a nurse [18]. These resignations cause financial loss to NQNs and the agency funding nurse education, loss of NQNs' confidence [19] negative effects on quality of care, and financial implications for staff recruitment.

Previous Taiwanese studies have largely focused on stress and transition with experienced nurses within Intensive Care Units and Emergency Departments, but not about transition to a NQN. Staff turnover studies are mainly quantitative [20], focused on attributes of the work environments [21] or used role stress models [12].

These provided limited opportunities to explore personal accounts and experiences of NQNs. The fact that $58 \%$ of NQNs leave within one year in Taiwan demonstrates the urgent need for research into experiences of Taiwanese NQNs in transition. NQNs' transition from the role of student to qualified nurse after being employed in their first posts in their nursing careers in Taiwan merits research.

\section{Methods}

\section{Aim of study}

The aim was to explore experiences of NQNs in Taiwan in transition to the role of a qualified nurse during the first twelve months of their career. The research questions were:

- What are the experiences of NQNs in their nursing role during the first twelve months following qualification?

- What factors influence NQNs adaptation to their role?

\section{Research design}

A cross-sectional design and exploratory qualitative research approach were selected to facilitate NQNs in expressing personal experiences, feelings and perceptions in exploring and understanding their experiences of transition using Bridges [8] "Managing Transition" as a framework.

\section{Sample}

Purposive sampling selected NQNs who had entered clinical practice during the previous twelve months and were willing to share their experiences. Data was finally collected from 28 participants in two teaching hospitals in Taiwan at three (Group 1), five (Group 2), and twelve months (Group 3) of employment.

Transition is a process which takes place over time. When change occurs, NQNs in the first three months after qualifying have to develop competence and adapt to their new role, but are unfamiliar with clinical tasks, procedures, and others expectations $[10,12,22]$. After three to five months, individuals beginning to face up to change/ the new situation find it difficult to know how best to cope with the new situation. Those emotions can develop into a personal transition crisis lasting a few weeks. A research highlighted that the process of transition from Rookie to Genuine nurses lasts six to twelve months [1]. However, previous studies were often conducted through interviews with small samples at one point of time. Furthermore, seldom have qualitative research methods been applied to consult NQNs to understand transition to their jobs in Taiwan. Therefore, this study aim to search the feelings and perspectives in different phases (three, five, and twelve months) of transition of NQNs.

\section{Data Collection}

Data were collected through in-depth, semi-structured interviews to explore NQNs' experiences. In addition, the interviewer, who are a qualitative researcher, had over ten years of work experience with the study topic. She has the ability to ask the participants' questions in a conversational format that seeks to explore the topic under investigation. Participants were interviewed at a place and time of their own choosing: all chose settings outside work. Face-to-face interviews (lasting 60 minutes) were digitally recorded. After the interview, the researcher gave a verbal summary to NQNs to help them validate the findings as real to their experience. A record of each interview and an outline of the process were noted, for reflection and interpretation [23]. Following transcriptions each participant was sent an email of their interview. All data related to all participants, including transcripts and digitally-audio recordings, were coded and stored until the end of this study.

\section{Data Analysis}

Data analysis used the Thematic Content Analysis framework (TCA) [23]. Verbatim interview transcripts were returned to interviewees for comment and correction (if necessary) to enhance rigour. Transcripts and recordings were scrutinized repeatedly to fully grasp the raw data. Headings were made as codes were induced; some meaningful category codes were generated and categorized into themes, and then systematically categorized to higher category themes. The researcher then returned to interview transcripts to highlight relevant paragraphs and collect meaningful quotations for reporting. Data analysis was undertaken by the researcher for each group, followed by further analysis across all groups to identify themes and sub-themes reflecting the first 12 months' experiences. An additional supervisor who is a qualitative researcher helped the researcher to confirm the data analysis in order to enhance the trustworthiness of the data.

\section{Results}

The study explores the 28 NQNs experiences of transition from student to nurse during their first twelve months after qualification in two selected hospitals in mid Taiwan. The demographic data of NQNs shows in Table 1.

\section{Theme 1: Perceptions of becoming a nurse}

Participants needed to perform their new role as NQNs and the first theme focused on what becoming a nurse meant for them. The three sub-themes identified were; becoming independent, joys under pressure, and praise for work well done.

Becoming independent nurses is an important issue as NQNs make the transition from students to staff nurses. After three months in clinical practice, all the NQN interviewees expressed both an awareness of their responsibility for patients and a realisation that they were now independent workers.

\section{This was a point highlighted by G1-7:}

"Once I became a real nurse I had a heightened sense of responsibility. I would think "Wow, I am a real nurse now. Many people are watching my every move, and I cannot conduct myself carelessly. I need to be accountable both to patients and to myself."

Once NQNs become nurses in practice, they face the nursing world and learn to survive. New nursing staff may receive either good or bad feedback from people in the practice situation. However, in addition to this new sense of responsibility, becoming an independent nurse often brings a sense of accomplishment. 


\begin{tabular}{|l|l|l|l|}
\hline NQNs & G1 & G2 & G3 \\
\hline Number & 8 & 12 & 8 \\
\hline Average age & 22 & 22 & 23 \\
\hline Gender & & & \\
\hline Male & 0 & 0 & 2 \\
\hline Female & 8 & 12 & 6 \\
\hline Months of experience & 3 & 5 & 12 \\
\hline Marital status & Single & Single & Single \\
\hline Education & & & \\
\hline Ass Bachelor & 5 & 8 & 6 \\
\hline Bachelor & 3 & 4 & 2 \\
\hline Work place & & & \\
\hline ICU & 2 & 1 & 3 \\
\hline General ward & 4 & 4 & 2 \\
\hline Other ward & 2 & 7 & 3 \\
\hline
\end{tabular}

Table 1 Summary of demographic data of NQNs

\section{This was highlighted by G1-2}

"I believe one of the biggest driving forces to continue in nursing is "having a sense of accomplishment." As I can gradually meet more of my own expectations in time at work, I begin to think I will continue in the nursing field.

At the same time, NQNs were no longer focused on their own confusion at work. They were paying more attention to the patients and NQNs also began to have slightly different perceptions of their nursing role. This was a point highlighted by half of participants, including G2-9: "Patients are my responsibility. I have the duty to care for them, so that their illnesses can improve, or their problems can be solved." NQNs in this stage were entering the stage of The New Beginning identified by Bridges (2003). He highlighted that nurses in The New Beginning can solve enough situations, and there is a rise in confidence in their role. This would continue until the end of about twelve months.

\section{This was highlighted by G3-2:}

"Some patients are more dependent on me. When a patient seeks my advice on everything, I think I must have done something well to make him ask me and value my opinions. It gives me a sense of achievement."

\section{Theme Two: Reality Challenge}

When nursing students go into clinical practice, they face a role change. The three sub-themes identified were: reality shock, caught off guard/stress, and work overload. As NQNs are now in the role of qualified nurses their clinical workload and responsibility are increased. All participants in this project identified their perceptions of conflict between the "real" world and the ideal.

\section{G1-6:}

"To me the job of nursing is synonymous with one other word, "busy." Nursing kept me so busy that I had no time to think about aspects of patient care. I only aimed to complete the doctor's orders." Dealing with sudden events and emergencies made for great stress for a majority of NQNs.

\section{G2A-9:}

"I would still be scared when emergencies happened. For example, if the patient required CPR, I felt so nervous, that I couldn't understand what the doctor said, and I was not sure what I was doing."
Work overload was described from the NQNs at the five month and the twelve month stages, resulting in loss of their enthusiasm for nursing. This was reported by G3-4:

"The stress is somewhat more than I can bear. For example, caring for too many patients affects the quality of care...., but time is limited; there are only eight hours. Even the most senior nurses are often delayed from getting off work on time."

\section{Theme Three: Developing Competence}

The three sub-themes identified were inadequate preparation/passing on experiences, inadequate clinical skills, and achievement of goals and learning complex care. When participants began their new role as staff nurses they needed to learn relevant skills and knowledge in order to develop competence. The evidence highlighted that graduate nurses felt a lack of professional knowledge and skills during the first year after being employed. This was highlighted by a number of NQNs, including G1-2:

"I felt there was too much to learn in too little time. I had too many things to consider while I was still unfamiliar with the work routines. Because I did not know where to begin I would feel irritated and disorganized."

\section{G1-8 reported her ideas:}

"I am still a bit scared about performing certain procedures. For example, it is hard for me to get the IV in on the first try. Even though I understand everything comes with experience, I am still afraid that I might harm patients because of my inexperience."

Inadequate ability in English affected NQNs' understanding of their patients' conditions as highlighted by a majority of participants in G1, including G1-1:

"A lot of the content of reports nowadays is in English, especially medical terminologies and data...I have to spend extra time looking up meanings of English words before I can hand over the shift. My poor English means I waste a lot of time."

In addition, NQNs needed further communication skills in their own language to provide care that was consistent with the patient's needs, especially when patients did not understand the illness, or their self management behaviours did not match professional suggestions they have been given by nurses. G1-2 described:

"Communicating with a patient's family was sometimes hard, 
especially when they did not understand the condition, and their opinions and perspectives did not match what I said."

Through the interviews in this study, in this situation NQNs identified that it was difficult to comfort the relatives and deal with their own emotions at the same time. Meanwhile, inadequate preparation of NQNs to meet the emotional needs of patients and their families, especially the bereaved, was apparent. This was mentioned by a number of NQNs, including.

\section{G1-1:}

"I did not know how to console the family when a patient passed away; I could only stand aside and watch them weep. That was also a great stress for me and I often needed time to adjust myself. I would tell myself not to think about it, and let time be the healer."

After five months, NQNs still thought that their knowledge was superficial and they needed to improve it. This was highlighted by all participants at this stage, including G2-7:

"As I still need to strengthen my academic knowledge I might consider going back to school to study some more after working for a full year and to continue to work while I am also studying."

NQNs' confidence increased during the first twelve months after becoming a nurse. NQNs at this stage had accumulated enough experience to catch the key point of patients' illnesses and complete exhaustive nursing assessments. This was highlighted by half of the NQNs in G3 and G3-2 offered:

"I had more confidence in providing patient care. I could grasp the main points of nursing and complete comprehensive assessments."

\section{Theme Four: Interpersonal Relationships}

This fourth theme focuses on the experiences of NQNs' interpersonal relationships with other staff members, including three sub-themes: learning to adjust to preceptors and senior nurses, developing interpersonal relationships, and balancing camaraderie and competition.

Newly qualified nurses needed to face not just a few preceptors but all nursing staff in their clinical area. NQNs cited that they had to adapt themselves to the many different style of doing things, as exemplified by G1-2:

"There were over 60 senior nurses in our ICU, and I had to get accustomed to each one's working style and thought process. It was quite difficult getting accustomed to the different senior nurses."

Furthermore, senior nurses criticising other colleagues also affected NQNs and they strove to keep away from such negative comments. As G1-2 stated:

"It was hard to hear others with similar seniority as me being criticized by a senior nurse. While I would try to do my best to avoid being criticized, I was afraid it may be inevitable."

Evidence in the present study indicated that interpersonal relationships affected NQNs' working and their interaction with senior nurses. This was offered by G2-6:

"If one's interpersonal relationships are poor work will not go smoothly. When there is a new admission every nurse will pitch in and help, but if a nurse's interpersonal relations are poor that person would have to manage without the help of others.

The need for effective interpersonal relationship among NQN's in the ward continued to be highlighted by participants at the end of their first year. All NQNs in G3 expressed that interpersonal relationships were important and affected their motivation at work.

\section{This was reinforced by G3-1:}

"My own mental ability and the encouragement from senior nurses are the reasons that I have come such a long way. I have learnt so much because they showed me my own shortcomings. I'd rather be scolded than not be told anything."
Furthermore, good support from among colleagues was identified as an important reason for new nurses to stay in nursing. G3-6 reinforced this view:

"The camaraderie among colleagues makes me want to stay. It would be hard for me to leave. Camaraderie is truly important."

\section{Discussion}

The findings have highlighted that participants identified the differences between clinical practice and nursing school when they experienced the "real" world of clinical practice as a NQN. The findings support Kramer [9] who used "reality shock" to describe the feelings of conflict between individuals' expectations and the reality of the clinical situations. The NQNs in this study experienced reality challenge during their first twelve months in practice. They felt there were many differences between what they learnt at nursing school and what happened in practice. When NQNs entered the workforce, they focused on completion of tasks, rather than performing patientcentred nursing as taught at nursing school. This finding corresponds with Halfer and Graf's [19] study that NQNs were concerned about getting their tasks done during the first six months after qualifying. However, NQNs realised that nursing not only involves patient care, but also associated complex tasks, resulting in a conflict between ideal and reality. Several situations including administrative work, preparation for clinical promotion, and perceived case overload were identified by NQNs at different stages of transition.

The transition from student to staff nurse role was perceived as a major change, particularly increased responsibility; previous studies highlighted that this can make NQNs feel stressed and anxious $[11,24]$. In this study, NQNs entering clinical practice quickly experienced increased responsibility which they found difficult to manage persisting during the first five months. Nursing workloads rapidly increased, with loss of their enthusiasm for nursing.

Many studies explore factors contributing to feelings of job satisfaction for NQNs [19, 25, 26, 27], but little reference is made in the literature to NQNs' experiences of losing enthusiasm for their work.

Experiences of NQNs entering clinical practice as registered nurses were not always negative, some participants identified that becoming an independent nurse brought a sense of accomplishment. NQNs may receive praise from senior nurses and appreciation from patients, positively enhancing their satisfaction at their performance. Responsibility can be two-sided; it may cause NQNs stress and anxiety, but also positively affect the progress of transition. After several months, NQNs could solve most clinical situations and get on well with senior nurses, resulting in increased confidence. NQNs at twelve months felt they could deal with almost all situations. NQNs at this point had the highest confidence in performing their role and began to look for a new purpose for their role. During the first twelve months employment, NQNs strove to fulfill their new role based on their knowledge and skills and their experiences in clinical practice during this period have been identified as reality challenge.

This study highlighted the two-sided nature of becoming a nurse, both the stress and achievements as NQNs progress through transition. The four key themes (Perceptions of becoming a nurse, Reality challenges, Developing competence, and Interpersonal relationships) incorporated both positive and negative issues. Personal and professional growth occurred during this year, with NQNs recognising the value of feedback from senior colleagues with growing awareness of their personal accountability, while working as an effective team member.

However, numerous challenges were identified. Many initial difficulties were considered due to limited preparation in clinical skills, team working and overall decision-making, English language and computer use, and no preparation for emergency-response. As time passed, participants focused more on social and work environment 
factors. They moved beyond their internal personal focus becoming aware of the wider work environment related to workload and social expectations of their role, over which they have little control. These issues and feelings of limited competence were associated with participants' thoughts about resignation, consistent with other findings that the work environment is a key determinant in such decisions [20,21].

According to the findings in the present study, NQNs at different stages presented distinct role performances. Figure 1 highlights the application of the themes and sub-themes which emerged from the interviews of NQNs at four stages in the progress of transition, in relation to Bridges' three phases. The NQNs at three months reflected that they tried to let to go of the dependency on preceptors, but they still needed suggestions and reminders from preceptors and senior nurses. The reality challenged them causing a variety of feelings when they performed their role, such as anxiety and fear. It reveals that NQNs at three month seemed to be staying in the phases of The Ending and The Neutral Zone. At five months, while NQNs felt in control of achieving their tasks, greater knowledge and skills were indispensable if the NQNs were to continue to improve their practice. The NQNs in this stage seemed to be within The Neutral Zone. The NQNs at twelve months were in renaissance and had begun to look for a new purpose for their role. As two nurses described, "I felt my role had become more important", "I have to be a model for the newest NQNs". These new purposes also brought emotional challenge such as fear and anxiety to the NQNs. This supports Bridges' (2003) report which stated that individuals at the New Beginning phase may suffer from emotional changes due to new demands which they thought they might not really understand.

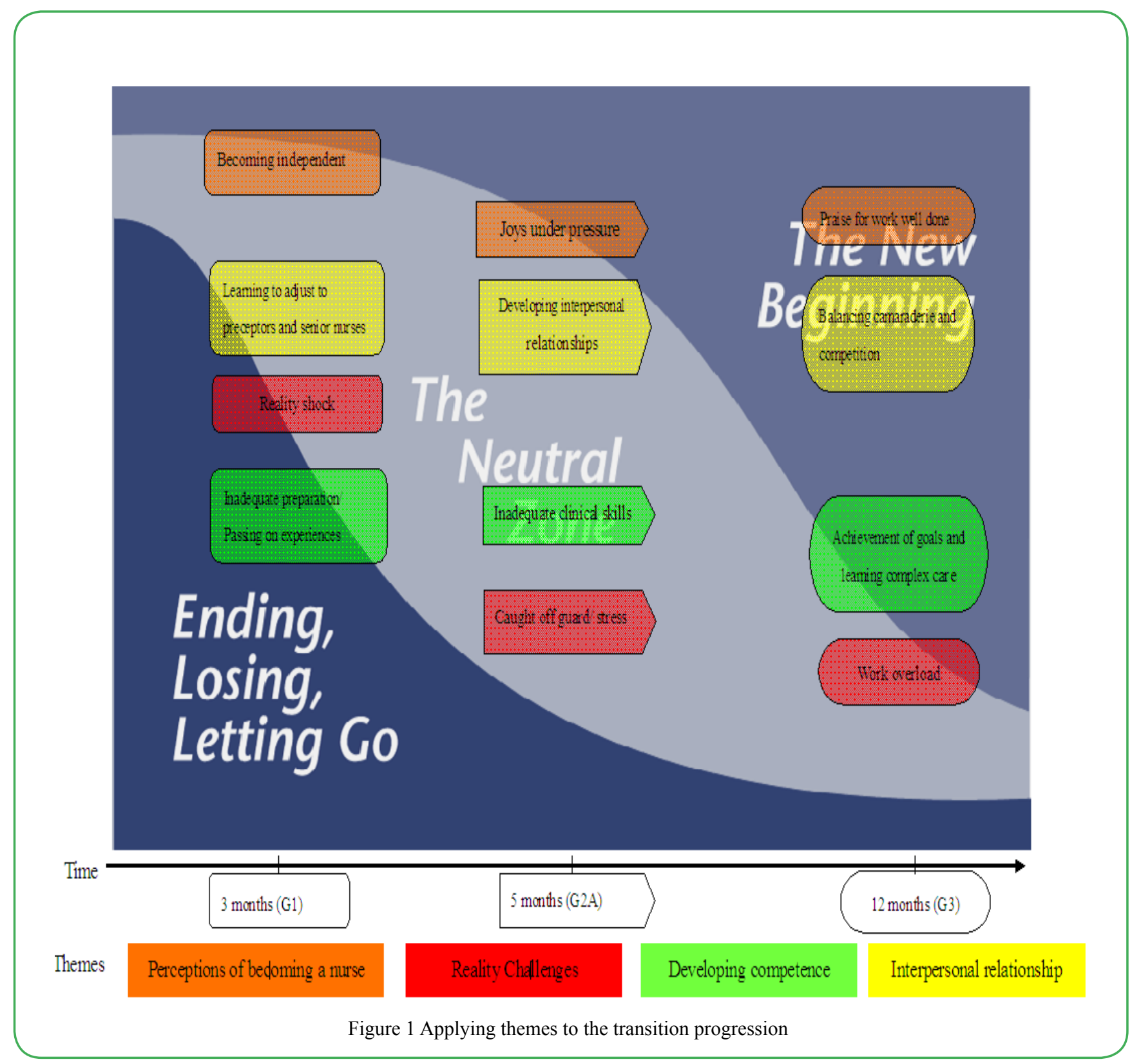




\section{Conclusion}

This study demonstrated that transition is complex involving time and development of clinical, organisational and decision-making skills. NQNs identified that their new role was based on what they evaluated as limited knowledge and skills. Their experiences in clinical practice during the first twelve months employment sound very like a reality challenge.

The findings have implications for education of student nurses and their support during transition to the role of qualified nurses. Chinese/English medical terminology training, nursing consulting and communication skills, nursing care associated with complex patients should be a component of the nursing curriculum as part of preparation of NQNs' competency. Otherwise, it is important that transition theory and concept should be a component of the Taiwan nursing curriculum in order to provide comprehensive understanding of transition to all nursing students, enabling them to undergo transition smoothly after being employed.

Hospitals claim that the current preceptorship programme (one to one attachment) is provided to NQNs for lasted three months. However, the NQNs in this study perceived that their preceptorship programme only lasted three to six weeks. One NQNs expressed that preceptors should focus on helping NQNs develop clinical competence and passing on their working experiences, rather than only guiding work routines. It is obvious that the role of preceptors is passing on their working experiences to NQNs and a well-designed preceptorship programme should be solidly grounded in practice. This requires a coordinated response from nursing schools, hospitals and government to increase those who adapt to their new roles and develop successful nursing careers.

\section{Acknowledgements}

We want to thank all the newly qualified nurses who, although in very different circumstances from our own, were willing, after work or during their time off, to allocate space to participate in our interviews. We are very grateful for all their help, and will always remember them with deep appreciation.

\section{Competing interest:}

We declare that we have no conflict of interest.

\section{References}

1. Andersson PL, Edberg AK (2010) The transition from rookie to genuine nurse: narratives from Swedish nurses 1 year after graduation. J Contin Educ Nurs 41: 186-92.

2. Duchscher JE (2009) Transition shock: the initial stage of role adaptation for newly graduated registered nurses. J Adv Nurs 65: 1103-13

3. Kralik D, Visentin K, van Loon A (2006) Transition: a literature review. J Adv Nurs 55: 320-329.

4. Bridges W (2003) Managing Transitions: making the most of change 2ed edition. Great Britain, Perseus 28.

5. Meleis AI, Sawyer LM, Im EO, et al (2000) Experiencing transitions: an emerging middle-range theory. ANS Adv Nurs Sci 23: 12-28.

6. Meleis AI (2007) Developing Middle Range and Situation Specific Theories: Exemplars, Theoretical Nursing Development and progress 4th edition, Lippincott, Williams \&Wilkins.

7. Williams D (1999) Human responses to change. Futures. 31: 609-616.

8. Bridges W (2003) Managing Transitions: making the most of change 2nd edition. Great Britain: Perseus.

9. Kramer M (1974) Reality shock. Why Nurses Leave Nursing? St Louis, MO: Mosby.
10. Duchscher JB (2008) A process of becoming: the stages of new nursing graduate professional role transition. J Contin Educ Nurs 39: 441-450.

11. O'Shea M, Kelly B (2007) The lived experiences of newly qualified nurses on clinical placement during the first six months following registration in the Republic of Ireland. J Clin Nurs 16: 1534-1542.

12. Chang E, Hancock K (2003) Role stress and role ambiguity in new nursing graduates in Australia. Nurs Health Sci 5:155-163.

13. Newton JM, McKenna L (2007) The transitional journey through the graduate year: a focus group study. Int J Nurs Stud 44: 1231-7.

14. Higgins G, Spencer RL, Kane R (2010) A systematic review of the experiences and perceptions of the newly qualified nurse in the United Kingdom. Nurse Educ Today 30: 499-508.

15. Wangensteen S, Johansson IS, Nordström G (2008) The first year as a graduate nurse--an experience of growth and development. J Clin Nurs 17: 1877-1885.

16. Maben J, Latter S, Clark JM (2006) The theory-practice gap: impact of professional-bureaucratic work conflict on newlyqualified nurses. J Adv Nurs.; 55: 465-477.

17. National Union of Nurses' Associations R.O.C (2016) Taiwan attends to active population.

18. Ministry of Labor (2016) A Study on Practice Environment and Physical and Mental Health of Nurses in Taiwan. Institute of Labor, Occupational Safety and Health, Ministry of Labor.

19. Halfer D, Graf E (2006) Graduate nurse perceptions of the work experience. Nurs Econ.; 24:150-152.

20. Chen HC, Chu CI, Wang YH, Lin LC (2008) Turnover factors revisited: a longitudinal study of Taiwan-based staff nurses. Int J Nurs Stud 45: 277-285.

21. Kramer M, Brewer BB, Maguire P (2013) Impact of healthy work environments on new graduate nurses' environmental reality shock. West J Nurs Res 35: 348-383.

22. Ellerton ML, Gregor F (2003) A study of transition: the new nurse graduate at 3 months. J Contin Educ Nurs 34: 103-107.

23. Newell R, Burnard P (2006) Research for evidence-based practice. Oxford, MO: Blackwell.

24. Moore C (2006) The transition from student to qualified nurse: a military perspective. Br J Nurs 7: 540-542.

25. Bowles C, Candela L (2005) First job experiences of recent RN graduates: improving the work environment. J Nurs Adm 35: 130-137.

26. Jackson C (2005) The experience of a good day: a phenomenological study to explain a good day as experienced by a newly qualified RN. Int J Nurs Stud 42: 85-95.

27. Murrells T, Robinson S, Griffiths P (2008) Job satisfaction trends during nurses' early career. BMC Nurs 7: 7. 